\title{
Stigma, Acceptance and Belonging for People with IDD Across Cultures
}

\author{
J. Jansen-van Vuuren ${ }^{1} \cdot$ H. M. Aldersey ${ }^{1}$ \\ Published online: 30 June 2020 \\ (C) Springer Nature Switzerland AG 2020
}

\begin{abstract}
Purpose of Review This review explores recent literature (from 2017 onwards) to identify current developments related to reducing stigma and increasing acceptance for people with Intellectual and Developmental Disabilities (IDD) in their communities across diverse cultures.

Recent Findings We identified literature related to experiences of stigma for people with IDD and their families across diverse cultures, as well as current and potential interventions and approaches for promoting acceptance.

Summary Although the literature confirms that stigma is still a major barrier to acceptance and inclusion for people with IDD regardless of culture, there appears to be progress in terms of using diverse approaches to support acceptance and belonging. Researchers are also increasingly acknowledging the importance of culture and context in the experience and mitigation of stigma. There remains a need for researchers and practitioners to include people with IDD in identifying and prioritising interventions that promote belonging within their communities.
\end{abstract}

Keywords Intellectual and developmental disability $\cdot$ Community integration $\cdot$ Culture $\cdot$ Diversity $\cdot$ Anti-stigma interventions . Belonging $\cdot$ Inclusion

\section{Introduction}

People with intellectual and developmental disabilities (IDDs) around the world experience stigma that can limit social inclusion and increase disparities with the general population [1]. Stigma involves discrimination, prejudice and exclusion of people in various forms [2] and often affects how one is accepted or can participate within a community [3]. It is complex and individual experiences can be highly varied and diverse, depending on the circumstances [4]. The literature describes various forms of stigma, including public stigma, selfstigma, courtesy stigma and affiliate stigma [5-7]. As indicated by its name, and most widely known, public stigma incorporates prejudicial, discriminatory and stereotypical perceptions and behaviours towards people with IDD from the

This article is part of the Topical Collection on Intellectual Disability

H. M. Aldersey

hma@queensu.ca

1 School of Rehabilitation Therapy, International Centre for the Advancement of Community Based Rehabilitation (ICACBR), Queen's University, Kingston, Canada broader society [5]. When individuals with IDD internalise these negative attitudes and behaviours towards them and believe themselves to be de-valued, this is referred to as selfstigma [6]. Conversely, courtesy stigma describes the prejudice or discrimination experienced by others associated with the person with IDD (i.e. family, friends), and affiliate stigma occurs when they also internalise these negative attributes and endorse such stereotypes prevalent in society [6, 7]. Stigma and exclusion can have serious consequences for people's participation, mental wellbeing and overall quality of life [8], hence, the importance of developing and implementing interventions to promote community acceptance and participation of people with IDD.

Researchers are also recognising that in many cases, cultural values, beliefs, and practices influence how stigma is expressed and experienced for people with IDD, as well as approaches for increasing community acceptance and belonging $[9 \cdot 10,11]$. For example, individuals with IDD and their families in low-income countries often appear to experience greater stigma with more serious consequences $[9 \bullet, 12 \bullet, 13 \bullet$, 14-16], including some children with IDD being chained and beaten in Ethiopia [15] or in Nigeria, certain people with IDD being abandoned and left homeless, and denied basic rights to 
education, healthcare and employment [16]. Ditchman et al. [17] discuss Triandis' four-factor conceptualisation of culture in relation to stigma towards people with IDD, including horizontal individualism and collectivism, and vertical individualism and collectivism, where vertical cultures value hierarchy whereas horizontal cultures value equality. Historically, Western values of individualism and promoting independence and productivity have predominated the framing of stigma and inclusion interventions [18, 19]; however, several authors critique this approach as perpetuating implicit prejudice against people with IDD $[18,20 \cdot$. Now, there appears to be increasing discussion about the importance of adapting anti-stigma interventions to be relevant within the specific cultural context. Consequently, for support providers from outside of the given cultural context of the individuals or communities they are serving, cultural training is critical to ensure their approaches are relevant and appropriate [21, 22].

In addition to cultural considerations for stigma reduction, recent findings from the IDD literature demonstrate that interventions aimed simply at service access or attendance and increasing social networks are insufficient, and we need to look beyond mere 'acceptance' as the end goal. Instead, people with IDD describe belonging as crucial for community acceptance, participation and overall wellbeing in various settings $[23 \bullet \bullet, 24 \bullet, 25,26]$. Renwick et al. [27•] depict the experience of belonging for youth with IDD as 'engaging in social relationships, interacting with people who are similar, negotiating meaningful roles in the community, and through navigating norms and expectations finding a good fit' (p. 951). The quality of relationships and interaction with others is more important than just being included or invited, and reciprocity and respect should characterise these relationships $[27 \bullet, 28 \bullet]$. Several Australian studies have found that people with IDD describe belonging '(i) in relation to place, (ii) as being part of a community, (iii) as having relationships and (iv) as identity' [28•] (p. 1095), and that '[b]eing known and valued is central to a secure sense of belonging and personal identity' [29] (p. 65). Belonging is a subjective, complex and multifaceted construct that puts greater focus on understanding the individual and their context and promoting agency/choice rather than implementing generic approaches to inclusion [28•]. Thus, incorporating community belonging as a critical outcome for anti-stigma interventions also resonates with the need to consider cultural values and nuances.

If community acceptance and belonging for people with IDD are end-goals for anti-stigma interventions, it is also important to consider the construct of 'community'. Boelé [20•] challenges some of the traditional assumptions of community, which can imply 'sameness' and 'normalisation' and where achieving access to a community relies on culturally bound values of independence, productivity and power imbalances. It is not enough for interventions to attempt to make the individual with IDD more 'acceptable' or conform to society (i.e. through increasing independent living skills) or even to simply remove some of the access barriers so that people can participate. She contends that we need to reconceptualise our idea of community and appreciate its complexities and tensions by focusing on justice, care, dignity and giving and embracing diversity: 'Full independence, a false ideal for anyone, should not be the end goal [for achieving community]... Community does not manifest when members achieve access to an ideology, group of people, or physical space, but, rather, when participants practice unconditional giving to each other' ( $p$. 399). Others similarly recognise that independence and productivity are not the only ways to participate in a community and there needs to be emphasis on 'ways of being' and realistic/feasible goals for people with IDD to engage with and belong in their community [18]. Hence, societallevel interventions also need to address some of these misconceptions around 'community' and aim to challenge oppressive attitudes and behaviours such that those with IDD and all people are accepted and valued for who they are. Therefore, this review will explore the recent literature to understand current approaches and developments to mitigate stigma and promote community acceptance and belonging for people with IDD across diverse cultures.

\section{Method}

The authors searched relevant databases including CINAHL, Embase, PsycInfo and Global Health, using keywords such as intellectual and developmental disability, learning disability, stigma, discrimination, prejudice, stereotype, acceptance, inclusion, community and culture. Due to the large number of articles identified and the breadth of relevant information, only articles published from 2017 onwards and those focusing specifically on approaches to reduce stigma or cultural nuances were included to identify the most recent developments. The first author conducted database searches, and both authors independently screened titles and abstracts before discussing selection of articles for full text inclusion. We included seventy-four full-text articles in the review and extracted information relating to stigma and acceptance (experiences and interventions), and cultural aspects, using a table. Through inductive analysis, the authors broadly categorised interventions according to individual, family and community-level interventions as well as interventions at multiple levels. We also identified themes and unique highlights related to cultural experiences of stigma and implications for promoting acceptance and belonging. 


\section{Addressing Stigma at Different Levels}

\section{Individual-Level Interventions}

Several researchers discuss interventions aimed at the individual level to minimise stigma and promote community acceptance and inclusion of individuals with IDD. More traditional approaches to empower individuals with IDD include selfadvocacy groups [30], and education on various components such as legal [31•] and financial rights [32•], as well as skills training. For example, several authors mention the importance of education around practical skills such as accessing public transport, money management, using assistive technology, social skills and personal characteristics (e.g. building self-esteem, self-identity and assertiveness) $[29,32 \bullet, 35 \bullet \cdot, 36]$, as well as work skills through career experience [36]. These initiatives for individual skill acquisition align with a more traditional approach of developing independence and supporting people with IDD to assimilate with societal norms and expectations, hence reducing their 'otherness' and enhancing their ability to function and participate within society.

\section{Family-Level Interventions}

In terms of family-level interventions to reduce stigma, education and empowerment of parents/caregivers are most frequently mentioned; these interventions aim to both decrease family's negative attitudes and behaviours towards IDD and to support them to adequately provide for their family's needs and cope with additional care-burdens, thus enhancing their acceptance as capable, contributing members of society. These interventions consist of education about IDD (including addressing real causes) $[12 \bullet, 37 \bullet, 38 \bullet \bullet]$, psychosocial wellbeing such as self-efficacy, stress management and coping strategies [9••, 39-41]; parental roles in supporting education and career prospects [14]; the influence of language [12•, 42]; creating a conducive home environment for children with IDD to develop and learn [43•]; crisis prevention and management [44]; behavioural interventions [45] and providing information on available services [38••, 41, 42]. The literature also highlights peer support and self-help associations for family members as important for mitigating stigma by raising awareness of IDD and providing emotional and practical support and connectedness, particularly in low-income countries where other government-led initiatives are minimal $[9,12]$. To identify practical interventions for promoting acceptance from parents' perspective, Louw and colleagues [46•] used an appreciative inquiry approach with South African families of adolescents with IDD; parents suggest that changing their routines and prioritising time together could facilitate family cohesion, inclusion and acceptance of the individual with IDD. In other low-income contexts, such as Namibia, family-targeted interventions can involve income-generation projects to combat poverty and empower families and individuals with IDD, reducing stigma and promoting community acceptance by demonstrating their contribution and capabilities rather than deficits and perceived burden [38••].

Cultural considerations can influence the focus of interventions. For example, many Asian and African countries have more collectivist cultures that value harmony, conformity and interdependence [17, 47]; as such, family-level interventions may be more culturally relevant so that families can model acceptance and inclusion [12•], strengthen traditional kinship networks [11] and promote sustainable interventions particularly in low-resource settings [45]. In relation to families of children with IDD in Thailand, Persons [43•] suggests that "[c]ultural activities strengthen family resilience and offer an alternative construction of meaning" (p. 121), for example through child-naming ceremonies which indicate acceptance and incorporation into the family. Therefore, there is a need for family-based interventions that promote inclusion in meaningful cultural activities.

Awareness of cultural values should also influence the way that family-level interventions are delivered. For example, many Arab families highly value privacy [48], and Chinese families are often more concerned with 'saving face' and avoiding shame [9••]; hence, support providers need to be particularly sensitive about maintaining confidentiality and not drawing attention to families of people with IDD (even unintentionally), recognising and reducing the barriers to help-seeking. Many Asian cultures also highly value academic success and families of children with IDD can be more susceptible to stigma and exclusion $[37 \cdot, 39]$; thus, educational interventions may be particularly important for these families. Interestingly, Durling et al. [49] describe how, although Bangladeshi families demonstrate less stigma by encouraging people with IDD to participate in the 'cycle of life' (i.e. marriage, parenthood), there is a tension related to individual autonomy and informed consent versus cultural values. Thus, interventions to promote community acceptance need to consider both individual needs and family values.

Gender is another critical consideration for interventions $[1,50]$, and gender differences are often more pronounced in certain cultures. At the family-level specifically, women (predominantly mothers) usually bear the greatest responsibility for supporting people with IDD, both children and adults, and can bear the brunt of stigma, for example in the Congo [12•], India [39], Kenya [51], South Africa [52••], Turkey [40] and in Arab cultures [48]. Additionally, Taderera and Hall [38••] highlight how, in Namibia, fathers often struggle to face the stigma of having a child with IDD, leaving mothers alone with the double stigma of being a single mother of a child with IDD. Some authors suggest that interventions need to specifically focus on the wellbeing and support of women caregivers, including culturally appropriate psychosocial counselling [9••, 40]. In contrast, Kabiyea and Manor-Binyamini [53] 
highlight how fathers in the patriarchal structure of a Bedouin Israeli community are 'central figures in the wide circle in which families raise adolescents with DD' (p. 37), and therefore may be particularly susceptible to stigma and require culturally appropriate support. Moving forward, interventions need to take a stronger gender-responsive approach to antistigma interventions, recognising dual disadvantage or multiple layers of stigma that may manifest for an individual or family. Men and women with IDD may face different types of stigma related to culturally constructed gender norms (e.g. not working in the community may not be as stigmatising for women if most women in the society do not work outside the home) and would require different forms of interventions.

\section{Societal-Level Interventions}

The majority of discussion around interventions to reduce stigma and promote acceptance of people with IDD focuses on targeting the broader society. At the government level, many researchers call for changes in policy to promote acceptance and protect people with IDD (i.e. from abuse and discrimination) in their communities [14, 16, 29, 32•, 52••, 54]. However, several authors highlight that even with policies in place, effective implementation is not occurring in practice $[16,55]$. Hamdani et al. [18] suggest that even positive public health policies such as the Canadian comprehensive mental health strategy - Open Minds, Healthy Minds - can perpetuate stigma and may need refinement to promote greater acceptance and inclusion for people with IDD through more feasible ways for people to belong in the community simply by 'being'.

Additionally, researchers discuss education aimed at various levels of society as a primary approach to mitigate stigma and promote acceptance. Firstly, educating society through anti-stigma campaigns and awareness raising is emphasised across diverse cultures, even if the approach differs in each context [13•, 16-18, 33, 38, 40, 44, 45, 48, 56, 57]. Several researchers suggest that collaborating with traditional forms of support (e.g. religious leaders with existing authority and respect) can improve public awareness and attitudes and enhance acceptance of people with IDD into their communities $[9 \cdot \bullet, 37 \bullet]$. Recognising the importance of religious beliefs for resilience and coping is particularly pertinent in some cultures and needs to be considered for effective intervention approaches $[9 \bullet \bullet, 46 \bullet]$. For example, Kang-Yi et al. [37•] demonstrate the influence of the church in the lives of Korean families of children with IDD and discuss how church-based outreach programmes and church leaders should have a role in education and supporting community acceptance. However, education and conscientising of such community and religious leaders is also necessary so that they can provide accurate information to communities and affirming support to families and individuals with IDD [38••]. Additionally, stigma is often amplified in contexts where causation of the IDD is attributed to spiritual or otherworldly forces (i.e. sorcery, parents' 'sin', punishment, test of faith) $[12 \bullet, 16,22,38 \bullet \bullet, 45,49,51,58 \bullet]$. In these contexts, community awareness programmes aimed at explaining real causes of IDD are highly relevant to reduce stigma and prejudice against individuals and families with IDD [16].

Media can also play an important role in mitigating stigma and changing societal attitudes towards IDD as Jamal [22] suggests in India. Consistent with education to change societal attitudes, Lindau and colleagues [59] demonstrate how a brief digital intervention can improve societal attitudes towards people with IDD, although direct contact and familiarity should also be encouraged [60]. Interestingly, Delgado and colleagues [61] highlight how community interventions to reduce stigma should not only address the more obvious negative reactions towards IDD but also affective reactions associated with 'compassion', as these emotions can perpetuate "paternalistic prejudice and the stereo-type of low competence and high sociability" (p. 120).

Education aimed at health and teaching professionals also appears to be an important component of anti-stigma interventions. Several researchers focus on school-aged people with IDD and identify the need for teacher education to effectively reduce stigma and promote inclusion, as teacher behaviour and language can significantly influence social acceptance of children with IDD [42, 62, 63]. Siperstein et al. [64] discuss the effectiveness of an American school campaign to mitigate stigma by focusing on reducing negative language use. They suggest that the campaign was successful because it was school-wide, peer-led and used direct education such as classroom activities and videos. In addition, researchers from diverse cultural contexts emphasise that health professionals and community health workers need ongoing education to improve attitudes and service provision towards people with IDD $[15,18,21,41,48,52,65,66]$, as unfortunately, research has shown little difference in stigmatising attitudes from professionals, despite their supposed knowledge and familiarity with IDD [22, 66].

As well as educational interventions, the literature highlights interventions aimed at facilitating community participation for people with IDD which can promote acceptance and belonging. For example, Kruithof et al. [67] describe a 'communal table' programme in the Netherlands that encourages social networks and inclusion through regularly sharing a meal together. However, participants had mixed responses and the authors conclude that simply gathering people together is not enough to create inclusion; rather, focusing interventions on peoples' existing social networks as well as combining with individually focused interventions may prove more effective. Similarly, several studies, also in the Netherlands, explore inclusion of people with IDD within their neighbourhoods and the authors suggest that different 
strategies are needed depending on the neighbouring 'pattern', but supporting people with IDD to perform social roles within their neighbourhood can enhance acceptance and inclusion $[68,69]$. Community recreational programmes are also important for facilitating acceptance and inclusion, but they must consider people with IDD's individual choices so that they can truly feel that they belong [70].

Interventions to promote community access for people with IDD are another important aspect for reducing stigma and promoting acceptance. Such interventions should address issues of physical accessibility such as public transport, appropriate signage and safety, as well as creating a socially accessible and inclusive environment [24•, 29, 34, 71]. Appropriate community-based housing can also promote acceptance and inclusion [32]. Miskimmin et al. [72] discuss facilitators for community inclusion specifically for older people, including housing close to essential services and ongoing support. They also recommend that centralised health services can improve inclusion and participation [72], and Taderera and Hall [38••] concur that unification of services would reduce stigma for families of children with IDD in Namibia by making services more accessible and mainstream, thus empowering families and improving public awareness and familiarity with IDD.

A number of researchers suggest workplace interventions to mitigate stigma and support acceptance and inclusion, for example, job coaching and integrated employment $[32 \bullet, 52 \bullet \bullet]$. Lysaght and colleagues [24•] identify several facilitators for workplace inclusion for people with IDD, including opportunities for regular, ongoing contact with others, shared workplace experiences, routines and events, supportive supervisors and co-workers and appropriate job-matching to promote strengths. In the USA, Raynor et al. [36] highlight the importance of 'building partnerships with employers' and 'building awareness and shar[ing] resources' to promote acceptance by facilitating employment opportunities for young people with IDD. Interestingly, there is some debate regarding workplace intervention approaches as several researchers recommend people with IDD should be encouraged to work in the open labour market $[31 \bullet, 32 \cdot]$, while others found that some people with IDD experience less stigma and prefer sheltered work options, as they feel safer and more accepted [24•]. Alternatively, Westoby and Shevellar [73] describe another option to sheltered workshops and mainstream workplaces, emphasising the benefits of community-based cooperatives that can provide greater social support and partnerships for people with IDD and promote a socially inclusive and meaningful work environment.

In addition to debates around best practice for workplace interventions, there appears to be ongoing tension in the literature around inclusive education across cultures and the role of inclusion either in promoting or reducing acceptance of people with IDD. Although the international push is for inclusive education, children with IDD are often not accepted into mainstream schools [22] and even in European countries, researchers have found that inclusive schooling in regular schools may not always increase acceptance [63]. 'Special' schools can provide a more comfortable and less stigmatising environment for children with IDD to interact with peers at a similar level; however, they can also perpetuate exclusion of children from the society [42]. Significantly, some researchers demonstrate that both special schools and inclusive schools can perpetuate stigma, for example through parents' and teachers' low expectations and negative language use [42, 64]. Chabeda et al. [51] found that parents of children with IDD in Kenya recommend that schools have a special needs unit and develop special education curriculum. In some countries, parents prefer to send their children to special schools as they feel their child is safer, less vulnerable to stigma and abuse, staff are more familiar with IDD [14] and education focuses more on life skills education not simply academic learning [43•]. Kassah, Kassah and Phillips [58•] discuss the tension between the benefits and limitations of special schools versus inclusive education in Ghana, suggesting we should 'acknowledge the holistic educational perspectives that some special schools in Ghana embrace' (p. 348) and that segregated schools are not meant to exclude children with IDD. They suggest there may be a place for both, as special schools can also encourage integration by networking with students from mainstream schools but on the familiar ground of children with IDD. It would be worthwhile to explore if these tensions exist in locations where best practices of inclusion are applied, as desires for special schools may in some instances be a response to inadequate attempts for inclusion (e.g. physically placing a student with IDD in a mainstream school without any support). An overall goal for truly inclusive schools should be that they value, recognise and support individual differences and community 'belonging', thereby reducing stigma.

\section{Addressing Stigma at Multiple Levels}

Although this literature review identifies stigma reduction and community inclusion interventions at specific levels (individual, family, society), the literature also reports interventions that cut across or incorporate multiple levels. For example, several researchers recommend mentoring as an approach at the individual and societal-level to promote acceptance, participation and inclusion [23••, 32•], including two USA-based peer-support programmes for school-to-work transitions [19], and university participation [74], and an intergenerational 'Men's Shed' mentoring programme in Australia [75]. Results show promise for building community networks, increasing public awareness and understanding about IDD including more positive attitudes and less sympathy/pity and improving competence and personal growth for both mentors 
and mentees, although further refinement and research is required. Additionally, a recent review exploring approaches to enhance social inclusion for young people with IDD highlights the emergence of social media and digital platforms for promoting participation and inclusion [35••]. These forms of stigma-reduction approaches may be increasingly more relevant in the COVID-19 and post-COVID-19 era, where evolving community norms for social distancing and social isolation may limit in-person interventions, in the short term and beyond. For example, social media and digital platforms may be used for actually delivering interventions at all levels that may have previously been delivered in person. Moreover, people with IDD who have challenges with mobility or experience anxiety or behavioural issues when out in the community may find increasing opportunities for interpersonal connections and engagement in community from the comfort and familiarity of their homes.

Arts-based programmes are also showing promise for reducing stigma across multiple levels, as Richards et al. [76] discuss a programme for men with IDD, where they used various art forms (e.g., sculpture, photography, poetry, drama) to creatively express aspects of their identity and challenge misconceptions about IDD, highlighting art as 'a valuable and empowering way in which to promote the voices of people with learning disabilities' (p. 223). In line with arts-based approaches, Dickinson and Hutchinson [77•] describe a British theatre company that promotes acceptance and inclusion of people with IDD and shows remarkably positive results for individuals with IDD, their families and the broader community, including increased individual self confidence, positive self-identity, and wellbeing, and heightened awareness, understanding and acceptance of IDD from families and community members. The authors emphasise that one of the reasons for its success is the company's focus on members' strengths. Similarly, music can promote acceptance, as Rickson and Warren [78] explored in a university music course in New Zealand. However, they also identify limitations and the need to encourage greater enrolment of nondisabled peers and other strategies to better integrate young people with IDD. Other researchers demonstrate the need for 'inclusive literacy' and the positive influence of reading for the social inclusion of young people with profound IDD [79]. In a novel approach to reduce stigma and improve social inclusion for people with IDD, some Australian researchers piloted a dog-walking programme-finding that the presence of a dog protects against negative factors, increases social encounters and recognition and boosts their confidence to engage in social encounters [80].

Given the link between poverty and stigma, researchers have identified that community-based interventions at all levels (individual, family, societal) are particularly important for stigma reduction and community integration in countries that face challenges of poverty, political and societal instability and limited resources to provide services $[13 \bullet, 15$, $38 \bullet, 52 \bullet, 55]$. For example, Tilahun et al. [15] demonstrate that training local non-specialist community health workers in Ethiopia can improve their attitudes to IDD, which is important for the provision of community-based rehabilitation (CBR) for individuals with IDD and their families in settings with limited health professionals. A scoping review of inclusive citizenship for people with IDD in South Africa [52••] identifies the importance of also considering issues of race and identity in stigma interventions and the need for "protective and participatory community-based services" (p. 12).

\section{Conclusion}

Despite the continued prevalence of stigma and exclusion for people with IDD and their families across cultures, their does appear to be a positive trend towards more strengths-based approaches for promoting acceptance and belonging for people with IDD [9••]. Additionally, the literature shows more involvement of people with IDD in research which is an encouraging direction, as regardless of the approach, people with IDD need to be included in developing interventions, recognising their expertise and respecting their choices and

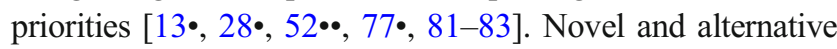
methods to anti-stigma interventions are also showing promise; however, further research is needed particularly with people with profound IDD [84] to understand and promote belonging and acceptance. Our review highlights the significant influence of culture on both experiences of stigma and approaches to promote acceptance and belonging. Hence, service providers need to recognise and respond to different cultural values and practices so that they can develop and provide appropriate and effective support to facilitate community acceptance and belonging for individuals with IDD and their families [9••]. However, it is critical to recognise that within any given culture, there will be significant nuances and differences among people. We suggest that future interventions strive to be culturally and contextually responsive by adapting to meet the needs of individuals, families and communities in a given context, time and place, rather than simply applying a broad 'culturally specific' brush for all. Understanding general cultural norms or values is an important starting point, but it is also critical to respond to the direct needs or preferences of each targeted person, family or community when intervening.

Our findings also demonstrate the need to develop interventions that promote 'belonging' for people with IDD, requiring a person-centred approach that incorporates individual needs and priorities, as well as broader cultural values [28•, 49]. The predominant Western focus on independence and productivity can in fact perpetuate stigma and exclusion for people with IDD, and it will be important for anti-stigma work moving forward to reconceptualise community so that it can 
become a place where people with IDD are accepted and belong, regardless of their ability and contribution. Individuals with IDD, families, communities and practitioners can all be involved in creating such communities that overcome stigma by embracing diversity and fostering acceptance and belonging for all.

\section{Compliance with Ethical Standards}

Conflict of Interest The authors declare that they have no conflicts of interest.

Human and Animal Rights and Informed Consent This article does not contain any studies with human or animal subjects performed by any of the authors.

\section{References}

Papers of particular interest, published recently, have been highlighted as:

- Of importance

•. Of major importance

1. Ditchman N, Werner S, Kosyluk K, Jones N, Elg B, Corrigan PW. Stigma and intellectual disability: potential application of mental illness research. Rehabil Psychol. 2013;58(2):206-16. https://doi. org/10.1037/a0032466.

2. Goffman E. Stigma: notes on the management of spoiled identity. New York: Simon and Schuster; 1963.

3. Scior K, Werner S, editors. Intellectual disability and stigma. UK: Palgrave Macmillan; 2016.

4. Link BG, Phelan JC. Conceptualizing stigma. Annu Rev Sociol. 2001;27(1):363-85. https://doi.org/10.1146/annurev.soc.27.1.363.

5. Werner S. Stigma in the area of intellectual disabilities: examining a conceptual model of public stigma. Am J Intellect Dev Disabil. 2015;120(5):460-75. https://doi.org/10.1352/1944-7558-120.5. 460.

6. Ali A, Hassiotis A, Strydom A, King M. Self stigma in people with intellectual disabilities and courtesy stigma in family carers: a systematic review. Res Dev Disabil. 2012;33(6):2122-40. https://doi. org/10.1016/j.ridd.2012.06.013.

7. Mak W, Cheung R. Affiliate stigma among caregivers of people with intellectual disability or mental illness. J Appl Res Intellect Disabil. 2008;21(6):532-45. https://doi.org/10.1111/j.1468-3148. 2008.00426.x.

8. Major B, Dovidio JF, Link BG, Calabrese SK. Stigma and its implications for health: introduction and overview. In: Major B, Dovidio JF, Link BG, editors. The Oxford handbook of stigma, discrimination, and health. New York: Oxford University Press; 2017.

9.• Mitter N, Ali A, Scior K. Stigma experienced by families of individuals with intellectual disabilities and autism: a systematic review. Res Dev Disabil. 2019;89:10-21. https://doi.org/10.1016/j. ridd.2019.03.001 This is a comprehensive systematic review of family experiences of stigma highlighting the cultural influence on stigma and the more positive trend towards parenting a child with IDD.

10. Scior K, Addai-Davis J, Kenyon M, Sheridan JC. Stigma, public awareness about intellectual disability and attitudes to inclusion among different ethnic groups. J Intellect Disabil Res.
2013;57(11):1014-26. https://doi.org/10.1111/j.1365-2788.2012. 01597.x.

11. Yang LH, Kleinman A, Link BG, Phelan JC, Lee S, Good B. Culture and stigma: adding moral experience to stigma theory. Soc Sci Med. 2007;64(7):1524-35. https://doi.org/10.1016/j. socscimed.2006.11.013.

12. Aldersey H, Kavira S, Kiasimbua J, Lokako W, Miaka P, Monte L. Stigma experienced by families with members with intellectual disabilities in Kinshasa, Democratic Republic of the Congo. Intervention. 2018;16(2):119-28. https://doi.org/10.4103/INTV. INTV $13 \_18$ This study uses a participatory approach and comprehensively discusses stigma in the DRC as well as potential areas for intervention.

13. Gona JK, Newton CR, Hartley S, Bunning K. Persons with disabilities as experts-by experience: using personal narratives to affect community attitudes in Kilifi, Kenya. BMC Int Health Hum Rights. 2018;18(1):18. https://doi.org/10.1186/s12914-018-0158-2 This study shows the impact of personal narratives to improve community attitudes as well as cultural nuances to stigma and stigma reduction.

14. Opoku MP, Nketsia W, Banye MA, Mprah WK, Dogbe JA, Badu E. Caregiving experiences and expectations of parents with inschool children with intellectual disability in Ghana. Res Dev Disabil. 2020;96:103524. https://doi.org/10.1016/j.ridd.2019. 103524

15. Tilahun D, Fekadu A, Tekola B, Araya M, Roth I, Davey B, et al. Ethiopian community health workers' beliefs and attitudes towards children with autism: impact of a brief training intervention. Autism. 2019;23(1):39-49. https://doi.org/10.1177/ 1362361317730298.

16. Sango PN. Country profile: intellectual and developmental disability in Nigeria. Tizard Learn Disabil Rev. 2017;22(2):87-93. https:// doi.org/10.1108/TLDR-07-2016-0019.

17. Ditchman N, Easton AB, Batchos E, Rafajko S, Shah N. The impact of culture on attitudes toward the sexuality of people with intellectual disabilities. Sex Disabil. 2017;35(2):245-60. https:// doi.org/10.1007/s11195-017-9484-x.

18. Hamdani Y, Ary A, Lunsky Y. Critical analysis of a population mental health strategy: effects on stigma for people with intellectual and developmental disabilities. J Ment Health Res Intellect Disabil. 2017;10(2):144-61. https://doi.org/10.1080/19315864.2017. 1281362.

19. Fisher MH, Athamanah LS, Sung C, Josol CK. Applying the selfdetermination theory to develop a school-to-work peer mentoring programme to promote social inclusion. J Appl Res Intellect Disabil. 2019;33(2):296-309. https://doi.org/10.1111/jar.12673.

20. Boelé A. In search of community: lessons from idealized independence for adults with disabilities. Harv Educ Rev. 2017;87(3):380 403. https://doi.org/10.17763/1943-5045-87.3.380 This article provides an interesting discussion around reconceptualising 'community'.

21. Barnoy S, Biton A, Itzhaki M. Social inclusion of children with down syndrome: Jewish and Muslim mothers' knowledge, attitudes, beliefs, and behavioral intentions. J Pediatr Nurs. 2017;35: 50-6. https://doi.org/10.1016/j.pedn.2017.02.035.

22. Jamal G. Stereotypes about adults with learning disabilities: are professionals a cut above the rest? Disabil CBR Inclusive Dev. 2019;30(2):7-36. https://doi.org/10.5463/dcid.v30i2.811.

23.• Bigby C, Anderson S, Cameron N. Identifying conceptualizations and theories of change embedded in interventions to facilitate community participation for people with intellectual disability: a scoping review. J Appl Res Intellect Disabil. 2018;31(2):165-80. https://doi.org/10.1111/jar.12390 Another comprehensive review that describes what community participation means for prople with IDD and intervention approaches. 
24. Lysaght R, Petner-Arrey J, Howell-Moneta A, Cobigo V. Inclusion through work and productivity for persons with intellectual and developmental disabilities. J Appl Res Intellect Disabil. 2017;30(5):922-35. https://doi.org/10.1111/jar.12284 This article gives detailed and practical approaches to facilitate inclusion in the workplace from the perspective of people with IDD.

25. Wilson NJ, Jaques H, Johnson A, Brotherton ML. From social exclusion to supported inclusion: adults with intellectual disability discuss their lived experiences of a structured social group. J Appl Res Intellect Disabil. 2017;30(5):847-58. https://doi.org/10.1111/ jar.12275.

26. Dimitrellou E, Hurry J. School belonging among young adolescents with SEMH and MLD: the link with their social relations and school inclusivity. Eur J Spec Needs Educ. 2019;34(3):312-26. https://doi.org/10.1080/08856257.2018.1501965.

27. Renwick R, Dubois D, Cowen J, Cameron D, Fudge Schormans A, Rose N. Voices of youths on engagement in community life: a theoretical framework of belonging. Disabil Soc. 2019;34(6):94571. https://doi.org/10.1080/09687599.2019.1583551 This article describes a framework of belonging based on findings from a participatory action research project with young people with IDD.

28. Strnadová I, Johnson K, Walmsley J. “... but if you're afraid of things, how are you meant to belong?" What belonging means to people with intellectual disabilities? J Appl Res Intellect Disabil. 2018;31(6):1091-102. https://doi.org/10.1111/jar.12469 This article also describes belonging from the perspective of people with IDD and emphasises a person-centred approach.

29. Robinson S, Hill M, Fisher KR, Graham A. Belonging and exclusion in the lives of young people with intellectual disability in small town communities. J Intellect Disabil. 2020;24(1):50-68. https:// doi.org/10.1177/1744629518765830.

30. Anderson S, Bigby C. Self-advocacy as a means to positive identities for people with intellectual disability: 'we just help them, be them really'. J Appl Res Intellect Disabil. 2017;30(1):109-20. https://doi.org/10.1111/jar.12223.

31. Byhlin S, Käcker P. 'I want to participate!' Young adults with mild to moderate intellectual disabilities: how to increase participation and improve attitudes. Scand J Disabil Res. 2018;20(1):172-81. https://doi.org/10.16993/sjdr.58 This article describes challenges and facilitators for participation for young people with IDD to support them as active citizens.

32. Heffron JL, Spassiani NA, Angell AM, Hammel J. Using photovoice as a participatory method to identify and strategize community participation with people with intellectual and developmental disabilities. Scand J Occup Ther. 2018;25(5):382-95. https://doi.org/10.1080/11038128.2018.1502350 This article uses photovoice to highlight environmental barriers and facilitators to participation for people with IDD.

33. Werner S, Abergel M. What's in a label? The stigmatizing effect of intellectual disability by any other name. Stigma Health. 2018;3(4): 385-94. https://doi.org/10.1037/sah0000108.

34. Hall SA. Community involvement of young adults with intellectual disabilities: their experiences and perspectives on inclusion. J Appl Res Intellect Disabil. 2017;30(5):859-71. https://doi.org/10.1111/ jar. 12276.

35.• Louw JS, Kirkpatrick B, Leader G. Enhancing social inclusion of young adults with intellectual disabilities: a systematic review of original empirical studies. J Appl Res Intellect Disabil. 2019. https://doi.org/10.1111/jar.12678 This is a thorough review identifying various intervention approaches to minimise stigma and enhance social inclusion.

36. Raynor O, Hayward K, Semenza G, Stoffmacher B. Community conversations to increase employment opportunities for young adults with developmental disabilities in California. J Disabil
Policy Stud. 2018;28(4):203-15. https://doi.org/10.1177/ 1044207317739405.

37. Kang-Yi CD, Grinker RR, Beidas R, Agha A, Russell R, Shah SB, et al. Influence of community-level cultural beliefs about autism on families' and professionals' care for children. Transcult Psychiatry. 2018;55(5):623-47. https://doi.org/10.1177/1363461518779831 This article describes the cultural aspects of stigma for Korean families particularly from the perspective of church leaders.

38.• Taderera C, Hall H. Challenges faced by parents of children with learning disabilities in Opuwo, Namibia. Afr J Disabil. 2017;6:e1e10. https://doi.org/10.4102/ajod.v6i0.283 This article clearly highlights cultural influences on stigma and acceptance, specifically within Namibia.

39. Banga G, Ghosh S. The impact of affiliate stigma on the psychological well-being of mothers of children with specific learning disabilities in India: the mediating role of subjective burden. J Appl Res Intellect Disabil. 2017;30(5):958-69. https://doi.org/10. 1111/jar.12311.

40. Duran S, Ergun S. The stigma perceived by parents of intellectual disability children: an interpretative phenomenological analysis study. Anadolu Psikiyatri Dergisi. 2018;19(4):390. https://doi.org/ 10.5455/apd.282536.

41. Werner S, Stern I, Roth D, Tenenbaum A. Help-seeking by parental caregivers of individuals with intellectual disabilities and dual diagnosis. Admin Pol Ment Health. 2019;46(3):321-33. https://doi. org/10.1007/s10488-018-00915-w.

42. O'Byrne C, Muldoon OT. The construction of intellectual disability by parents and teachers. Disabil Soc. 2019;34(1):46-67. https://doi. org/10.1080/09687599.2018.1509769.

43. Persons DS. Stigma, learning and inheritance: an ecocultural study of adaptation and resource use among families of children with Down syndrome in Thailand. Disabil CBR Inclus Dev. 2017;28(2):117-28. https://doi.org/10.5463/dcid.v28i2.597 Another very interesting discussion around cultural influences on stigma, highlighting the benefit of inclusion for people with IDD in cultural activities.

44. Spassiani N, Abou Chacra MS, Lunsky Y. "Why are you here? Can't you cope at home?" The psychiatric crisis of people with intellectual disabilities and the community's response. J Ment Health Res Intellect Disabil. 2017;10(2):74-92. https://doi.org/10. 1080/19315864.2016.1278290.

45. Thoresen SH, Fielding A, Gillieatt S, Blundell B, Nguyen L. A snapshot of intellectual disabilities in Lao PDR: challenges for the development of services. J Intellect Disabil. 2017;21(3):203-19. https://doi.org/10.1177/1744629517704535.

46. Louw CJ, Grobler HB, Cowden RG. Strengthening intellectually challenged adolescents' sense of self: an appreciative inquiry mixed-methods intervention. J Interdisc Health Sciences. 2018;23(9):e1-e11. https://doi.org/10.4102/hsag.v23i0.1113 This article describes a strengths-based approach - appreciative inquiry - and mentions practical strategies for including adolescents with IDD into their families and community.

47. Chang YC, Chen CH, Huang PC, Lin LY. Understanding the characteristics of friendship quality, activity participation, and emotional well-being in Taiwanese adolescents with autism spectrum disorder. Scand J Occup Ther. 2019;26(6):452-62. https://doi.org/10. 1080/11038128.2018.1449887.

48. Habayeb S, Dababnah S, John A, Rich B. Cultural experiences of Arab American caregivers raising children with autism spectrum disorder. J Autism Dev Disord. 2020;50(1):51-62. https://doi.org/ 10.1007/s10803-019-04218-3.

49. Durling E, Chinn D, Scior K. Family and community in the lives of UK Bangladeshi parents with intellectual disabilities. J Appl Res Intellect Disabil. 2018;31(6):1133-43. https://doi.org/10.1111/jar. 12473. 
50. Morán M, Gómez L, Alcedo M, Pedrosa I. Gender differences in social inclusion of youth with autism and intellectual disability. J Autism Dev Disord. 2019;49(7):2980-9. https://doi.org/10.1007/ s10803-019-04030-z.

51. Chabeda-Barthe J, Wambua T, Chege WL, Hwaga D, Gakuo T, Rotich GC. Child developmental disabilities, caregivers' role in Kenya and its implications on global migration. Int J Environ Res Public Health. 2019;16(6):1-20. https://doi.org/10.3390/ ijerph16061010.

52.• Capri C, Abrahams L, Mckenzie J, Coetzee O, Mkabile S, Saptouw $\mathrm{M}$, et al. Intellectual disability rights and inclusive citizenship in South Africa: what can a scoping review tell us? Afr J Disabil. 2018;7(5):e1-e17. https://doi.org/10.4102/ajod.v7i0.396 An interesting review on the rights of people with IDD and discussion around cultural influences.

53. Kabiyea F, Manor-Binyamini I. The relationship between stress and stigma, somatization and parental self-efficacy among fathers of adolescents with developmental disabilities in the Bedouin community in Israel. Res Dev Disabil. 2019;90:31-40. https://doi.org/10. 1016/j.ridd.2019.04.004.

54. Wołowicz-Ruszkowska A, McConnell D. The experience of adult children of mothers with intellectual disability: a qualitative retrospective study from Poland. J Appl Res Intellect Disabil. 2017;30(3):482-91. https://doi.org/10.1111/jar.1232251.

55. Gevorgianiene V, Sumskiene E. P.S. for post-soviet: a glimpse to a life of persons with intellectual disabilities. J Intellect Disabil. 2017;21(3):235-47. https://doi.org/10.1177/1744629517701561.

56. Bachmann CJ, Höfer J, Kamp-Becker I, Küpper C, Poustka L, Roepke S, et al. Internalised stigma in adults with autism: a German multi-center survey. Psychiatry Res. 2019;276:94-9. https://doi.org/10.1016/j.psychres.2019.04.023.

57. Power A, Bartlett R. I shouldn't be living there because I am a sponger': negotiating everyday geographies by people with learning disabilities. Disabil Soc. 2018;33(4):562-78. https://doi.org/10. 1080/09687599.2018.1436039.

58. Kassah BLL, Kassah AK, Phillips D. Children with intellectual disabilities and special school education in Ghana. Int J Disabil Dev Educ. 2018;65(3):341-54. https://doi.org/10.1080/ 1034912X.2017.1374358 This article provides an interesting discussion around the tensions of inclusive education versus special schools in the Ghanaian context.

59. Lindau N, Amin T, Zambon A, Scior K. The effect of brief digital interventions on attitudes to intellectual disability: results from a pilot study. J Appl Res Intellect Disabil. 2018;31(1):106-13. https://doi.org/10.1111/jar.12366.

60. Werner S. Can interpersonal contact help improve attitudes toward soldiers with intellectual disability? An Israeli study. J Intellect Disabil Res. 2017;61(12):1162-73. https://doi.org/10.1111/jir. 12439.

61. Delgado N, Arino E, Betancor V, Rodriguez-Perez A. Intergroup trust and anxiety: the two sides of stigma towards people with Down syndrome. Anales De Psicologia. 2018;34(1):117-22. https://doi.org/10.6018/analesps.34.1.267311.

62. Avcioglu H. Classroom teachers' behaviors and peers' acceptance of students in inclusive classrooms. Educ Sci Theory Pract. 2017;17(2):463-92. https://doi.org/10.12738/estp.2017.2.0034.

63. Huber C, Gerullis A, Gebhardt M, Schwab S. The impact of social referencing on social acceptance of children with disabilities and migrant background: an experimental study in primary school settings. Eur J Spec Needs Educ. 2018;33(2):269-85. https://doi.org/ $10.1080 / 08856257.2018 .1424778$.

64. Siperstein GN, Albert AB, Jacobs HE, Osborne KJ, Stokes JE. A schoolwide approach to promoting student bystander behavior in response to the use of the word "retard". Res Dev Disabil. 2018;80: 142-52. https://doi.org/10.1016/j.ridd.2018.06.016.
65. Breau LM, Aston M, Macleod E. Education creates comfort and challenges stigma towards children with intellectual disabilities. J Intellect Disabil. 2018;22(1):18-32. https://doi.org/10.1177/ 1744629516667892.

66. Pelleboer-Gunnink HA, Van Oorsouw WMWJ, Van Weeghel J, Embregts PJCM. Mainstream health professionals' stigmatising attitudes towards people with intellectual disabilities: a systematic review. J Intellect Disabil Res. 2017;61(5):411-34. https://doi.org/ 10.1111/jir.12353.

67. Kruithof K, Suurmond J, Harting J. Eating together as a social network intervention for people with mild intellectual disabilities: a theory-based evaluation. Int J Qual Stud Health Well-being. 2018;13(1). https://doi.org/10.1080/17482631.2018.1516089.

68. Overmars-Marx T, Pepping B, Thomése F. Living apart (or) together-neighbours' views and experiences on their relationships with neighbours with and without intellectual disabilities. J Appl Res Intellect Disabil. 2018;31(6):1008-20. https://doi.org/10.1111/jar. 12455.

69. Overmars-Marx T, Thomése F, Meininger H. Neighbourhood social inclusion from the perspective of people with intellectual disabilities: relevant themes identified with the use of photovoice. J Appl Res Intellect Disabil. 2019;32(1):82-93. https://doi.org/10. 1111/jar.12511.

70. Merrells J, Buchanan A, Waters R. The experience of social inclusion for people with intellectual disability within community recreational programs: a systematic review. J Intellect Develop Disabil. 2018;43(4):381-91. https://doi.org/10.3109/13668250.2017. 1310822.

71. Melbøe L, Ytterhus B. Disability leisure: in what kind of activities, and when and how do youths with intellectual disabilities participate? Scand J Disabil Res. 2017;19(3):245-55. https://doi.org/10. 1080/15017419.2016.1264467.

72. Miskimmin C, Shooshtari S, Menec V, Duncan KA, Martin T, Stoesz BM. Age-friendly communities for older persons with intellectual disabilities. Qual Ageing Older Adults. 2019;20(4):206-18. https://doi.org/10.1108/QAOA-11-2018-0058.

73. Westoby P, Shevellar L. The possibility of cooperatives: a vital contributor in creating meaningful work for people with disabilities. Disabil Soc. 2019;34(9-10):1613-36. https://doi.org/10.1080/ 09687599.2019.1594699.

74. Harrison AJ, Bisson JB, Laws CB. Impact of an inclusive postsecondary education program on implicit and explicit attitudes toward intellectual disability. Intellect Dev Disabil. 2019;57(4):323-36. https://doi.org/10.1352/1934-9556-57.4.323.

75. Wilson NJ, Cordier R, Ciccarelli M, Maccallum J, Milbourn B, Vaz $\mathrm{S}$, et al. Intergenerational mentoring at men's sheds: a feasibility study. J Appl Res Intellect Disabil. 2018;31(1):e105-e17. https:// doi.org/10.1111/jar.12338.

76. Richards M, Lawthom R, Runswick-Cole K. Community-based arts research for people with learning disabilities: challenging misconceptions about learning disabilities. Disabil Soc. 2019;34(2): 204-27. https://doi.org/10.1080/09687599.2018.1522243.

77. Dickinson D, Hutchinson N. Changes in understandings and perceptions of individuals, significant others and community supporters involved in a theatre company for adults with intellectual disabilities. J Appl Res Intellect Disabil. 2019;32(3):691-705. https://doi.org/10.1111/jar.12564 An interesting article about the successful approach of a theatre company to increase community acceptance and belonging for people with IDD.

78. Rickson D, Warren P. Music for all: including young people with intellectual disability in a university environment. J Intellect Disabil. 2018;22(3):279-93. https://doi.org/10.1177/ 1744629517701860 .

79. Robinson D, Moore N, Harris C. The impact of books on social inclusion and development and well-being among children and young people with severe and profound learning disabilities: 
recognising the unrecognised cohort. Br J Learn Disabil. 2019;47(2):91-104. https://doi.org/10.1111/bld.12262.

80. Bould E, Bigby C, Bennett PC, Howell TJ. 'More people talk to you when you have a dog' - dogs as catalysts for social inclusion of people with intellectual disabilities. J Intellect Disabil Res. 2018;62(10):833-41. https://doi.org/10.1111/jir.12538.

81. Merrells J, Buchanan A, Waters R. "We feel left out": experiences of social inclusion from the perspective of young adults with intellectual disability. J Intellect Develop Disabil. 2019;44(1):13-22. https://doi.org/10.3109/13668250.2017.1283684.

82. Stanley Z, Lauretani P, Conforti D, Cowen J, Dubois D, Renwick $\mathrm{R}$. Working to make research inclusive: perspectives on being members of the Voices of Youths project. Disabil Soc. 2019;34(9-10):1660-7. https://doi.org/10.1080/09687599.2019. 1619232.

83. Mueller C. Adolescent understandings of disability labels and social stigma in school. Int J Qual Stud Educ. 2019;32(3):263-81. https://doi.org/10.1080/09518398.2019.1576940.

84. Gauthier-Boudreault C, Beaudoin AJ, Gallagher F, Couture M. Scoping review of social participation of individuals with profound intellectual disability in adulthood: what can I do once I finish school? J Intellect Develop Disabil. 2019;44(2):248-60. https:// doi.org/10.3109/13668250.2017.1310810.

\section{Key Points}

- Identifying what it means to belong is important for individuals and families of people with IDD so that barriers to acceptance can be reduced and facilitators strengthened.

- Caregivers and families play a crucial role in modelling acceptance and belonging for individuals with IDD, but they often face additional challenges and would benefit from contextually appropriate support (i.e. emotional, social, material, informational).

- Professionals working with individuals with IDD and their families need to consider broader cultural values as well as individual nuances and priorities to provide appropriate support and promote acceptance and belonging.

- As a broader society, we need to reconsider what it means to be a community and how we can foster acceptance and belonging through embracing and supporting diversity, individual strengths and everyone's inherent worth.

Publisher's Note Springer Nature remains neutral with regard to jurisdictional claims in published maps and institutional affiliations. 